\title{
Design of Low-Pass Type Inverter: UWB Band-Pass Filter with Low Spurious Characteristics
}

\author{
Young-Ho Cho $\cdot$ Moon-Gyu Choi $\cdot$ Sang-Won Yun
}

\begin{abstract}
In this paper, we present the design method for a low-pass type inverter, which can effectively suppress the spurious response associated with band-pass filters. The inverter has a length of $\lambda / 4$ and employs not only a stepped-impedance configuration but also asymmetrical and bending structures in order to improve frequency selectivity and compactness. The inverter is applied as an impedance/admittance inverter to the ultra-wideband (UWB) band-pass filter. The UWB band-pass filter configuration is based on a stub band-pass filter consisting of quarter-wavelength impedance inverters and shunt short-circuited stubs $\lambda / 4$ in length. The asymmetrical stepped-impedance low-pass type inverter improves not only the spurious responses, but also the return loss characteristics associated with a UWB band-pass filter, while a compact size is maintained. The UWB band-pass filter using the proposed inverters is fabricated and tested. The measured results show excellent attenuation characteristics at out-band frequencies, which exceed $18 \mathrm{~dB}$ up to $39 \mathrm{GHz}$. The insertion loss within the pass-band (from 3.1 to $10.6 \mathrm{GHz}$ ) is below $1.7 \mathrm{~dB}$, the return loss is below $10 \mathrm{~dB}$, and the group delay is below 1 ns.
\end{abstract}

Key words : Stepped-Impedance Low-Pass Type Inverter, Ultra-Wideband, Quarter-Wave Stubs, Wideband Filter.

\section{I . Introduction}

Bandpass filters with excellent out-of-band rejection and high selectivity are essential components of a wireless communication system. The wide stop-band characteristics of band-pass filters are usually required to be in association with the nonlinear components so as to eliminate the undesired interference, noise, and harmonics in the stop-band [1].

Several approaches have been introduced to enhance the spurious responses [2] [8]. One of the conventional ways is to push up the first spurious response to a higher frequency range by using stepped-impedance resonators [2]. In [3] and [4] attempts were made to minimize the difference between the even/odd mode velocities, or to equalize the modal electrical lengths of microstrip coupled lines to eliminate the spurious response. In [5], the implementation of the wiggly-line filter was introduced in order to suppress the first spurious passband. For a broader stop-band, the employment of a uniplanar compact photonic-bandgap (PBG) structure in the microstrip band-pass filter was also introduced in [6]. In addition, two independent transmission zeros can be created at the required frequencies in order to cancel the spurious responses by means of proper tapping at both the input and output resonators [7]. In [8], a band-pass filter with a wide-stop-band was proposed by using different types of resonators with the same fundamental resonant frequency, but with different higher-order resonant frequencies. However, these approaches have limitations in the elimination of the spurious responses. The most effective and simplest approach is to cascade a low-pass filter into a band-pass filter, which results in the degradation of the insertion loss characteristics and an increase in the filter size [9]. However, when the low-pass filter is employed as impedance/admittance inverters, the drawbacks of the previous designs can be overcome. In this paper, we present an analysis and design method for an asymmetrical stepped-impedance low-pass type inverter with an overall electrical length of $\lambda / 4$. The proposed inverter employs the asymmetrical and bending structure applied to the conventional stepped-impedance low-pass filter, so that it not only improves the performances of the spurious response and high selectivity, it does so while maintaining a compact size. Even though the proposed low-pass type inverter can be applied as impedance/admittance inverters on the band-pass filters, the UWB band-pass filter is chosen in order to show the usefulness of this type of inverter. The designed UWB filter is based on a stub band-pass filter which consists of quarter-wavelength transformers and quarter-wavelength shunt short-circuited stubs [10]. The configuration has the advantages of easy fabrication

Manuscript received December 16, 2010 ; revised March 17, 2011. (ID No. 20101216-041J)

Department of Electronic Engineering, Sogang University, Seoul, Korea.

Corresponding Author : Sang-Won Yun (e-mail : swyun@sogang.ac.kr) 


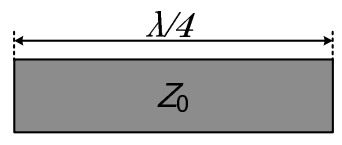

(a)

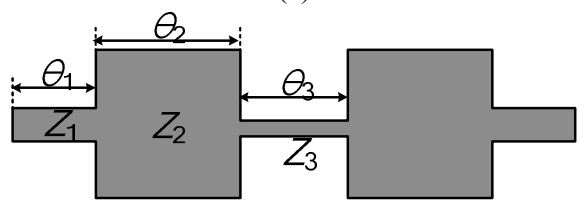

(b)

Fig. 1. (a) A conventional quarter-wavelength transformer. (b) The 5th-order stepped-impedance low-pass type inverter.

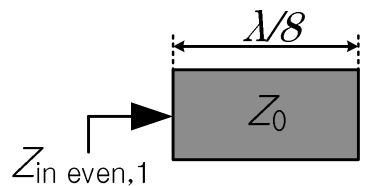

(a)

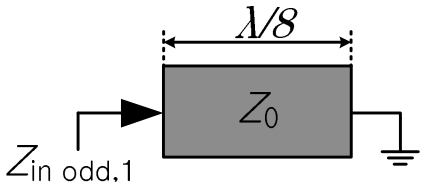

(b)
Fig. 2. (a) The even-mode equivalent circuit. (b) The oddmode equivalent circuit in Fig. 1(a).

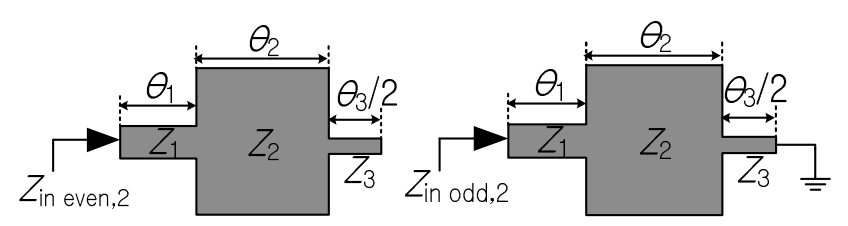

(a)

(b)

Fig. 3. (a) The even-mode equivalent circuit. (b) The oddmode equivalent circuit in Fig. 1(b).

and compact size.

This paper is organized as follows. In section 2 the analysis and design methods of the stepped-impedance inverter will be discussed. By using the even- and the odd-mode analysis approach, the precise design of the 5th-order stepped-impedance low-pass type inverter is performed. The UWB band-pass filter that is based on the stub band-pass filter is introduced briefly in section 3 and it is extended to adopt the proposed inverter. In section 4 , the simulated as well as the measured results of the UWB band-pass filter are discussed. Finally, the conclusions are presented in section 5 .

\section{II . A Study of the Asymmetrical Stepped-Impedance Low-Pass Type Inverter}

\section{2-1 Analysis of a Stepped-Impedance Low-Pass Type Inverter}

The design of the 5th-order stepped-impedance low-pass type inverter with an overall electrical length of $\lambda / 4$ can be achieved using an even/odd mode analysis. Since any higher-order low-pass type inverter of the Chebyshev and Butterworth responses can be analyzed in a similar manner, a 5th-order inverter of the Chebyshev response is chosen and will be analyzed for application to the UWB band-pass filter in section 3 .

Fig. 1 shows the $\lambda / 4$ transformer and the 5th-order stepped-impedance low-pass type inverter. In order to apply this type of inverter to the design of band-pass filter, the structure in Fig. 1(b) should have the same electrical length and characteristic impedance as the $\lambda / 4$ transformer in Fig. 1(a) within the frequency range of interest. Fig.2 and Fig. 3 show the even/odd mode equivalent circuits in Fig. 1(a) and (b), respectively.

When the conditions, $Z_{\text {in_even, } 1}=Z_{\text {in_even, } 2}$ and $Z_{\text {in_odd }, 1}=$ $Z_{\text {in_odd,2 }}$ are imposed, the following equations can be derived

$$
\begin{gathered}
-\frac{Z_{0}}{Z_{1}}=\frac{Z_{2}\left(Z_{3} \tan \frac{\theta 3}{2}+Z_{2} \tan \theta_{2}\right)+Z_{1} \tan \theta_{1}\left(Z_{2}-Z_{3} \tan \frac{\theta 3}{2} \tan \theta_{2}\right)}{Z_{1}\left(Z_{2}-Z_{3} \tan \frac{\theta 3}{2} \tan \theta_{2}\right)+Z_{2} \tan \theta_{1}\left(Z_{3} \tan \frac{\theta 3}{2}+Z_{2} \tan \theta_{2}\right)} \\
\frac{Z_{0}}{Z_{1}}=\frac{Z_{2}\left(Z_{3} \cot \frac{\theta 3}{2}+Z_{2} \tan \theta_{2}\right)+Z_{1} \tan \theta_{1}\left(Z_{2}-Z_{3} \cot \frac{\theta 3}{2} \tan \theta_{2}\right)}{Z_{1}\left(Z_{2}-Z_{3} \cot \frac{\theta 3}{2} \tan \theta_{2}\right)+Z_{2} \tan \theta_{1}\left(Z_{3} \cot \frac{\theta 3}{2}+Z_{2} \tan \theta_{2}\right)} .
\end{gathered}
$$

Since the configuration in Fig. 1(b) should have characteristics of conventional low-pass filters such as a Butterworth or Chebyshev low-pass filter response, the relationship between the phases $\left(\theta_{1}, \theta_{2}\right.$, and $\left.\theta_{3}\right)$ and the characteristic impedances $\left(Z_{1}, Z_{2}\right.$, and $\left.Z_{3}\right)$ in Fig. 1(b) should be satisfied as follows [16]:

$$
\begin{aligned}
& Z_{1}=\frac{f_{0} g_{1} Z_{0}}{f_{C} \theta 1} \\
& Z_{2}=\frac{f_{C} \theta 2 Z_{0}}{g_{2} f_{0}} \\
& Z_{3}=\frac{f_{0} g_{3} Z_{0}}{f_{C} \theta 3} .
\end{aligned}
$$

where $f_{\mathrm{C}}$ is the cut-off frequency of the low-pass type inverter in Fig. 1(b), $f_{0}$ is the center frequency of the $\lambda$ $/ 4$ transformer in Fig. 1(a), $g_{1}, g_{2}$ and $g_{3}$ are element values of the low-pass prototype filter, and $Z_{0}$ is the characteristic impedance of the $\lambda / 4$ transformer in Fig. 1(a).

Since equations (1) and (2) have the same magnitude, with the opposite sign, the summation of two equations becomes zero. Therefore, from equations (1) (5), the design equation can be obtained as 


$$
\begin{aligned}
& \frac{f_{C} \theta_{2} Z_{0}}{g_{2} f_{0}}\left(\frac{f_{0} g_{3} Z_{0}}{f_{C} \theta_{3}} \tan \frac{\theta_{3}}{2}+Z_{2} \tan \theta_{2}\right)+\frac{f_{0} g_{1} Z_{0}}{f_{C} \theta 1} \tan \theta_{1}\left(\frac{f_{C} \theta_{2} Z_{0}}{g_{2} f_{0}}-\frac{f_{0} g_{3} Z_{0}}{f_{C} \theta_{3}} \tan \frac{\theta 3}{2} \tan \theta_{2}\right) \\
& \frac{f_{0} g_{1} Z_{0}}{f_{C} \theta_{1}}\left(\frac{f_{C} \theta_{2} Z_{0}}{g_{2} f_{0}}-\frac{f_{0} g_{3} Z_{0}}{f_{C} \theta_{3}} \tan \frac{\theta_{3}}{2} \tan \theta_{2}\right)+\frac{f_{C} \theta_{2} Z_{0}}{g_{2} f_{0}} \tan \theta_{1}\left(\frac{f_{0} g_{3} Z_{0}}{f_{C} \theta_{3}} \tan \frac{\theta_{3}}{2}+\frac{f_{C} \theta_{2} Z_{0}}{g_{2} f_{0}} \tan \theta_{2}\right) \\
& =-\frac{\frac{f_{C} \theta_{2} Z_{0}}{g_{2} f_{0}}\left(\frac{f_{0} g_{3} Z_{0}}{f_{C} \theta_{3}} \cot \frac{\theta_{3}}{2}+\frac{f_{C} \theta_{2} Z_{0}}{g_{2} f_{0}} \tan \theta_{2}\right)+\frac{f_{0} g_{1} Z_{0}}{f_{C} \theta_{1}} \tan \theta_{1}\left(\frac{f_{C} \theta_{2} Z_{0}}{g_{2} f_{0}}-\frac{f_{0} g_{3} Z_{0}}{f_{C} \theta_{3}} \cot \frac{\theta 3}{2} \tan \theta_{2}\right)}{\frac{f_{0} g_{1} Z_{0}}{f_{C} \theta 1}\left(\frac{f_{C} \theta_{2} Z_{0}}{g_{2} f_{0}}-\frac{f_{0} g_{3} Z_{0}}{f_{C} \theta_{3}} \cot \frac{\theta_{3}}{2} \tan \theta_{2}\right)+\frac{f_{C} \theta_{2} Z_{0}}{g_{2} f_{0}} \tan \theta_{1}\left(\frac{f_{0} g_{3} Z_{0}}{f_{C} \theta_{3}} \cot \frac{\theta_{3}}{2}+\frac{f_{C} \theta_{2} Z_{0}}{g_{2} f_{0}} \tan \theta_{2}\right)} .
\end{aligned}
$$

To design the stepped-impedance low-pass type inverter with an electrical length of $\pi / 2$ and a characteristic impedance of $40 \Omega$ at the center frequency of 6.85 $\mathrm{GHz}$, the design parameters $\left(Z_{1}, Z_{2}\right.$, and $Z_{3}, \theta_{1}, \theta_{2}$, and $\theta_{3}$ ) in Fig. 1(b) can be obtained by using (1) (6). When $\theta_{2} / \theta_{1}$ is $\alpha$, (1) and (6) become equations only consisting of $\theta_{1}$ and $\theta_{3}$. Therefore, $\theta_{1}$ and $\theta_{3}$ can be obtained from (1) and (6). The value of $\theta_{2}$ can be subsequently determined as $\alpha \theta_{1}$. Finally, $Z_{1}, Z_{2}$ and $Z_{3}$ can be derived from (1) (3). Fig. 4 shows the impedance and phase variations for the configuration in Fig. 1(b) as a function of the $\alpha$ value $\left(=\theta_{2} / \theta_{1}\right)$. From Fig. 4, one can choose the feasible impedances and phases in Fig. 1(b) by selecting $\alpha$. Fig. 5 shows the magnitude and the phase characteristics of the $\lambda / 4$ transformer and the low-pass type inverter in Fig. 1 when $\alpha=2.5$. In this case, the impedances and the phases in Fig. 1(b) are chosen as follows: $Z_{1}=105 \Omega, Z_{2}=21 \Omega, Z_{3}=130 \Omega, \theta_{1}$ $=7^{\circ}, \theta_{2}=19^{\circ}$ and $\theta_{3}=13^{\circ}$. As shown in Fig. 5, the magnitude and the phase responses of the low-pass type inverter in the pass-band are similar to those of the $\lambda / 4$ transformer. Moreover, the attenuation characteristics are maintained at the stop-band above $15 \mathrm{GHz}$ in the lowpass type inverter configuration as shown in Fig. 5(a). Therefore, the stepped-impedance low-pass type inverter has an overall electrical length of $\lambda / 4$ at the center frequency of the pass-band, as well as possessing Chebyshev low-pass filter characteristics.

\section{2-2 Asymmetrical Stepped-Impedance Low-Pass Type Inverter with Enhanced Stop-Band Performances}

Asymmetrical stepped-impedance low-pass filters offer the advantage of an enhanced spurious response [17]. Fig. 6(b) is the equivalent circuit of the asymmetrical stepped-impedance configuration in Fig. 6(a) [18]. In Fig. 6(b), $R_{\mathrm{P} 1}$ and $R_{\mathrm{P} 2}$ are the series resistance from the radiation loss of the asymmetrical step discontinuity in Fig. 6(a). In [18], as the offset $(t)$ in Fig. 1(a) is increased, the loss by $R_{\mathrm{P} 1}$ and $R_{\mathrm{P} 2}$ is also increased, exponentially, with frequency. Accordingly, an asymmetrical stepped-impedance structure has effectively suppressed the spurious response in the out-band [17]. Moreover, the

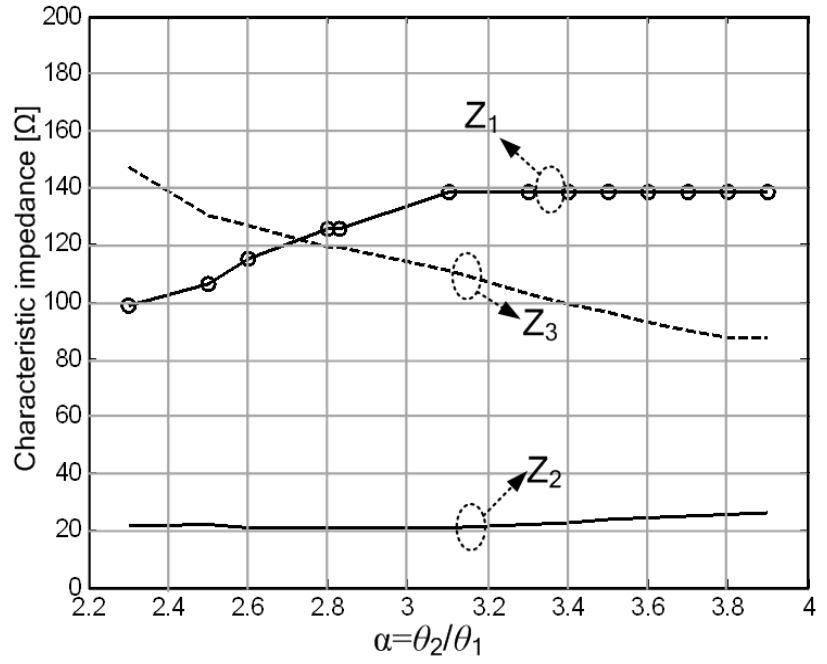

(a)

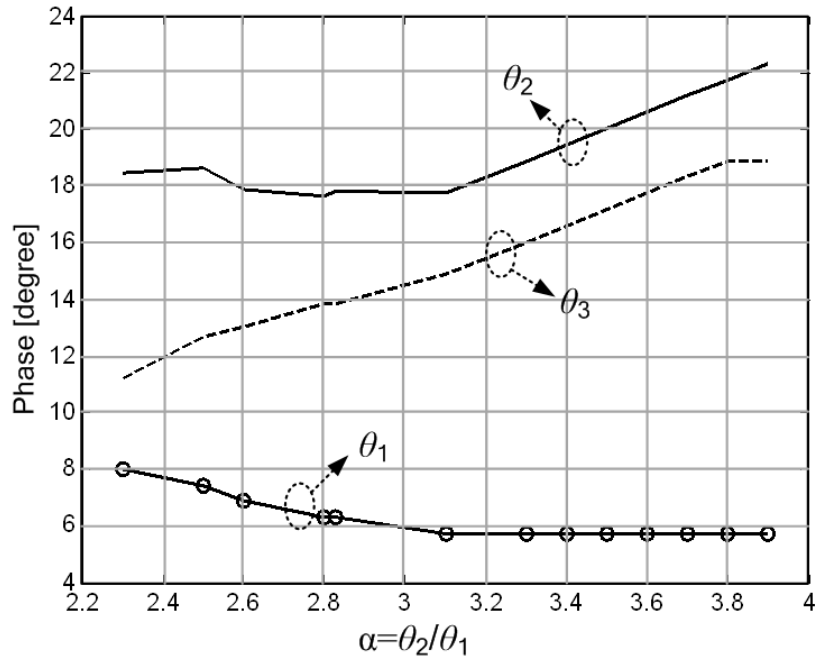

(b)

Fig. 4. (a) Variation of impedances in Fig. 1(b). (b) Variation of phases in Fig. 1(b) as a function of values of $\alpha\left(=\theta_{2} / \theta_{1}\right) .\left(f_{0}=6.85 \mathrm{GHz}, f_{C}=15 \mathrm{GHz}, Z_{0}=40\right.$ $\Omega, g_{1}=0.7563, g_{2}=1.3049$, and $g_{3}=1.5773$ (5th-order Chebyshev filter with $0.01 \mathrm{~dB}$ ripples).

asymmetrical stepped-impedance low-pass type inverter has transmission zeros in the out-band. Fig. 7(a) shows the stepped-impedance low-pass type inverter to which the asymmetrical configuration is applied. 


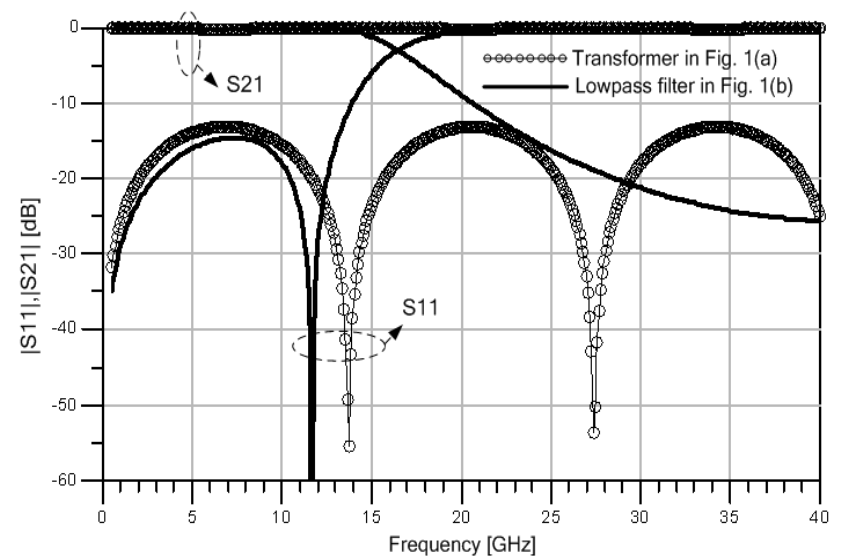

(a)

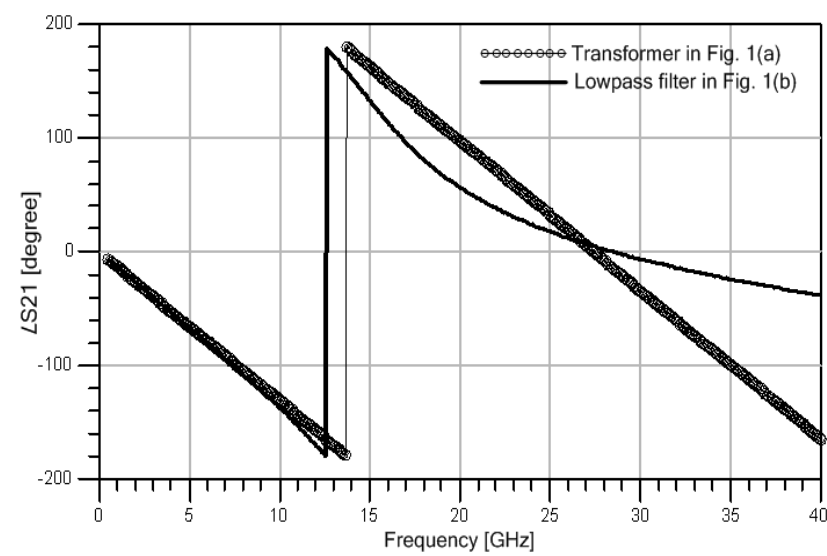

(b)

Fig. 5. (a) A simulated magnitude. (b) Simulated phase characteristics of the $\lambda / 4$ transformer $(6.85 \mathrm{GHz})$ with $40 \Omega$ characteristic impedance as well as the steppedimpedance lowpass type inverter $\left(f_{C}=15 \mathrm{GHz}\right)$ in Fig. $1\left(Z_{1}=105 \Omega, Z_{2}=21 \Omega, Z_{3}=130 \Omega, \theta_{1}=7^{\circ}, \theta_{2}\right.$ $=19^{\circ}$, and $\theta_{3}=13^{\circ}$ when $\alpha=2.5$ ).

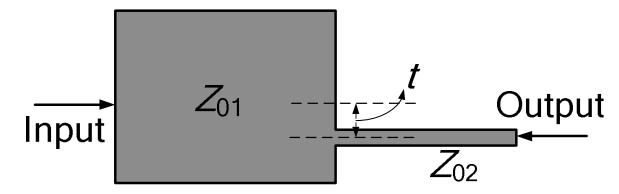

(a)

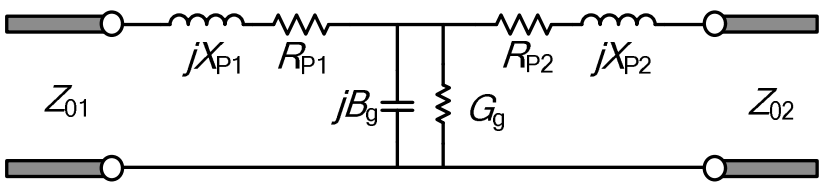

(b)

Fig. 6. (a) Stepped-impedance structure in which asymmetrical discontinuity is employed. (b) Equivalent circuit.

Fig. 7(b) and (c) are the simulation results of the conventional stepped-impedance low-pass type inverter and the asymmetrical stepped-impedance low-pass type in-

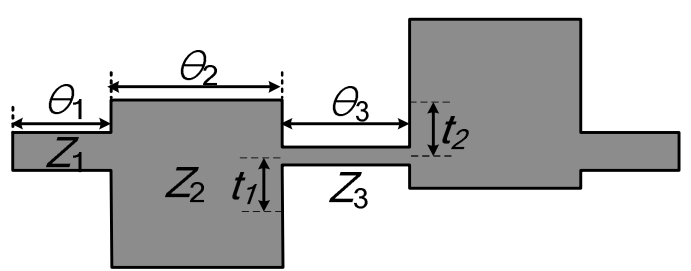

(a)

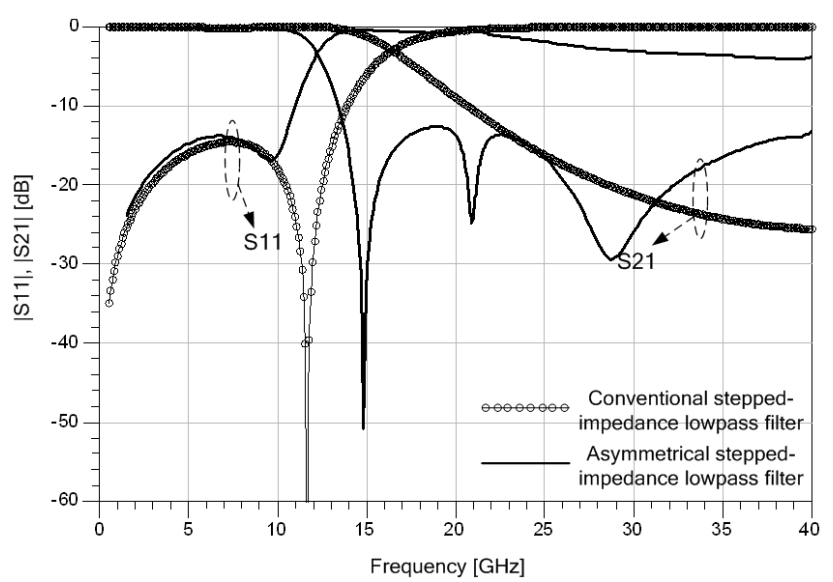

(b)

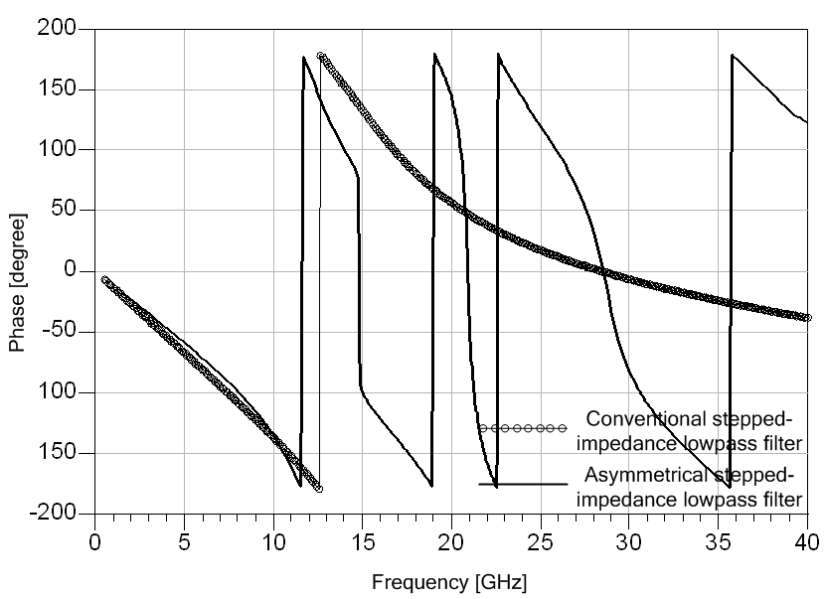

(c)

Fig. 7. (a) Asymmetrical stepped-impedance low-pass type inverter $\left(t_{1}=0.35 \mathrm{~mm}, t_{2}=1.2 \mathrm{~mm}, Z_{1}=105 \Omega, Z_{2}=\right.$ $21 \Omega, Z_{3}=130 \Omega, \theta_{1}=7^{\circ}, \theta_{2}=19^{\circ}$, and $\theta_{3}=13^{\circ}$ $\left.\left(f_{C}=15 \mathrm{GHz}\right)\right)$. (b) Simulated magnitude response. (c) Simulated phase response.

verter. The simulation is performed using Agilent's ADS Momentum. As shown in Fig. 7(b), the asymmetricalstepped-impedance low-pass type inverter has transmission zeros, because the low impedance section in Fig. 7(a) operates as an open stub in contrast to that of the conventional stepped-impedance low-pass type inverter in Fig. 1(b). Therefore, when asymmetrical step discontinuity is applied to the stepped-impedance low-pass type inverter, the spurious response is improved by the radiation loss $\left(R_{\mathrm{P} 1}\right.$ and $\left.R_{\mathrm{P} 2}\right)$ and the transmission zeros. 


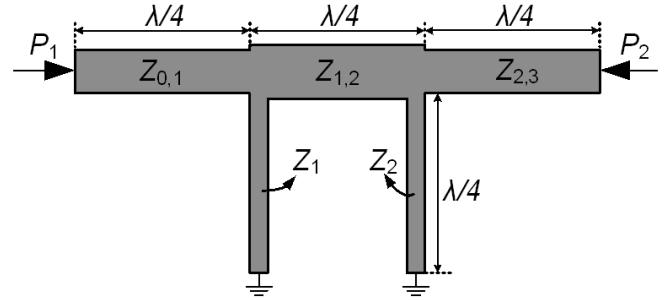

(a)

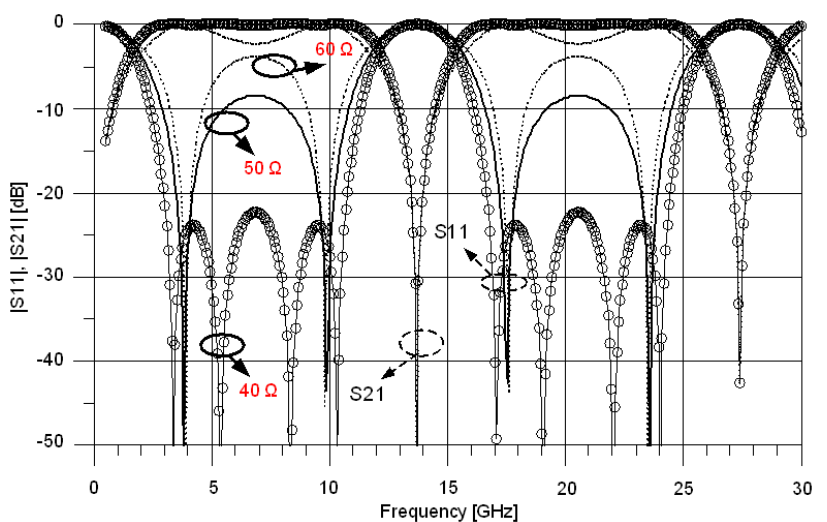

(b)

Fig. 8. (a) The layout of the 2nd-order stub UWB bandpass filter with $\lambda / 4$ impedance/admittance inverters and $\lambda / 4$ short-circuited stubs, and (b) its simulated responses for various values of $Z_{0,1}$ and $Z_{2,3}$.

\section{A Stub UWB Band-Pass Filter with a Proposed Low-Pass Type Inverter Acting as Impedance/ Admittance Inverters}

\section{3-1 A Stub UWB Band-Pass Filter}

The layout of the designed two-pole band-pass filter is shown in Fig. 8 (a). The filter consists of short-circuited shunt stubs and a $\lambda / 4$ inverter for compact size as in [16]. The filter is designed based on the following equations:

$$
\begin{aligned}
& \theta=\frac{\pi}{2}\left(1-\frac{F B W}{2}\right), \quad h=2 \\
& \frac{J_{1,2}}{Y_{0}}=g_{0} \sqrt{\frac{h g_{1}}{g_{2}}}, \frac{J_{n-1, n}}{Y_{0}}=g_{0} \sqrt{\frac{h g_{1} g_{n+1}}{g_{2}}} \\
& N_{i, i+1}=\sqrt{\left(\frac{J_{i, i+1}}{Y_{0}}\right)^{2}+\left(\frac{h g_{0} g_{1} \tan \theta}{2}\right)^{2}} \\
& Y_{1}=g_{0} Y_{0}\left(1-\frac{h}{2}\right) g_{1} \tan \theta+Y_{0}\left(N_{1,2}-\frac{J_{1,2}}{Y_{0}}\right) \\
& Y_{n}=Y_{0}\left(g_{n} g_{n+1}-g_{0} g_{1} \frac{h}{2}\right) \tan \theta+Y_{0}\left(N_{n-1, n}-\frac{J_{n-1, n}}{Y_{0}}\right) .
\end{aligned}
$$

In equation $(7) \sim(11)$, the value of $Y_{0}$ is filter admittance of the two-pole band-pass filter, and the $Y_{i} s \quad(i=1$, $2 \cdots n$ ) are the admittance value of the $\lambda / 4$ shunt stubs, and $J_{1,2}$ is the value of the $J$-inverter.

In order to design the UWB band-pass, the required parameters are $f_{0}=6.85 \mathrm{GHz}$, fractional bandwidth (FBW) $=1.09$ and $Y_{0}=0.02$. The element values for $0.01 \mathrm{~dB}$ ripple Chebyshev response are used. By using these element values and the design equations (7) (11), the impedance values for the layout can be derived as follows: $Z_{1}=101 \Omega, Z_{1,2}=33.7 \Omega$, and $Z_{0,1}=50 \Omega$. The simulated results can be obtained as shown in Fig. 8(b). This figure shows that the UWB band-pass filter has a bandwidth ranging from $3.1 \mathrm{GHz}$ to $10.6 \mathrm{GHz}$. The return loss within the pass-band can be enhanced by adjusting the value of $Z_{0,1}$ in Fig. 8(a). The number of poles increases when the impedance value of $Z_{0,1}$ is lower than $50 \Omega$. Therefore, the return loss characteristic is improved by using the lower impedance at the position of $Z_{0,1}[11]$.

\section{3-2 UWB Band-Pass Filter using the Proposed Inverter as the Input/Output Inverters Stub UWB Band-Pass Filter}

Fig. 9(a) shows the proposed UWB band-pass filter with the proposed asymmetrical stepped-impedance low-pass type inverter and Fig. 9(b) shows the simulated results. The simulation is performed using Agilent's ADS Momentum. The asymmetrical stepped-impedance lowpass type inverter is utilized as the impedance/admittance inverter instead of the $\lambda / 4$ transformer $\left(Z_{0,1}, Z_{2,3}\right)$ in Fig. 8 (a). To replace the $\lambda / 4$ transformers, the low- pass type inverter is designed using the values presented in Fig. 4, so that the low-pass type inverter has an overall electrical length of $\lambda / 4$ and a characteristic impedance of $40 \Omega$ at the $6.85 \mathrm{GHz}$ as shown in Fig. 7. For the compact size, a bending structure is applied to the low-pass type inverter in Fig. 7(a) as shown in Fig. 9(a). The overall size of the designed UWB band- pass filter is $23.1 \times 14.1 \mathrm{~mm}$.

\section{Measurement Results}

Fig. 10 shows the photograph of the fabricated UWB band-pass filter with the asymmetrical stepped-impedance low-pass type inverter. The substrate is Rogers RO3003 with a relative dielectric constant of 3.0 , a thickness of $20 \mathrm{mil}$, and a $\tan \delta=0.0013$. The results were measured using an Agilent $8510 \mathrm{C}$ vector network analyzer and Inter-Continental Microwave's substrate test fixture. The measured frequency responses are given in Fig. 11(a) and 11(b). As can be seen, the simulated and 


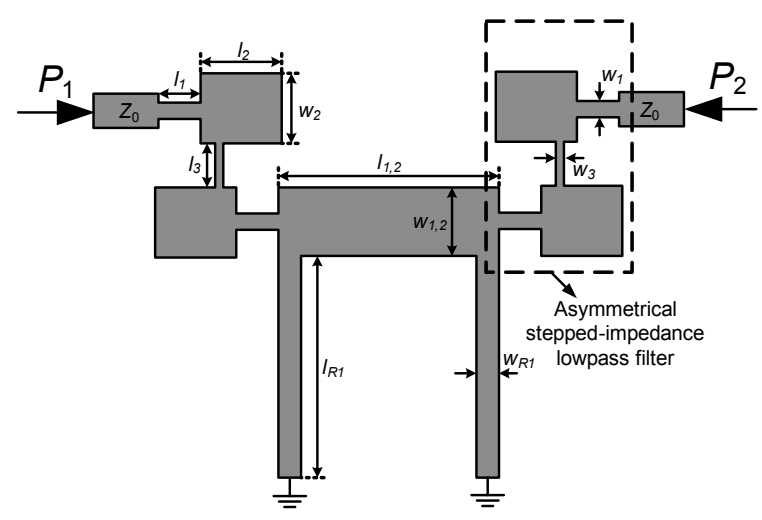

(a)

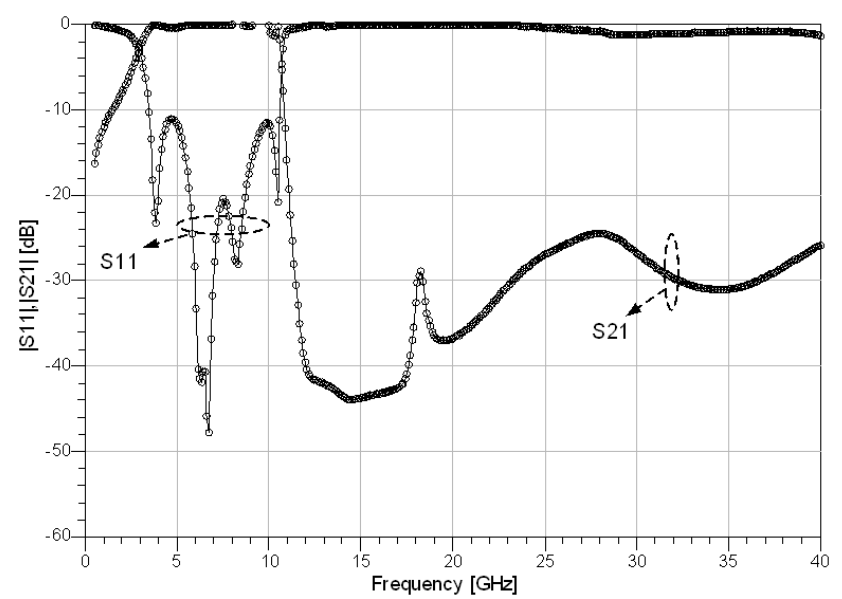

(b)

Fig. 9. (a) The layout of the proposed UWB bandpass filter with an asymmetrical stepped-impedance lowpass type inverter, and (b) Simulated response ( $l_{1}=3.4 \mathrm{~mm}, l_{2}=1.7$ $\mathrm{mm}, l_{3}=2.5 \mathrm{~mm}, l_{1,2}=7.4 \mathrm{~mm}, l_{\mathrm{R} 1}=8 \mathrm{~mm}, w_{1}=0.5$ $\mathrm{mm}, w_{2}=1.7 \mathrm{~mm}, w_{3}=0.1 \mathrm{~mm}$, and $\left.w_{\mathrm{R} 1}=0.5 \mathrm{~mm}\right)$.

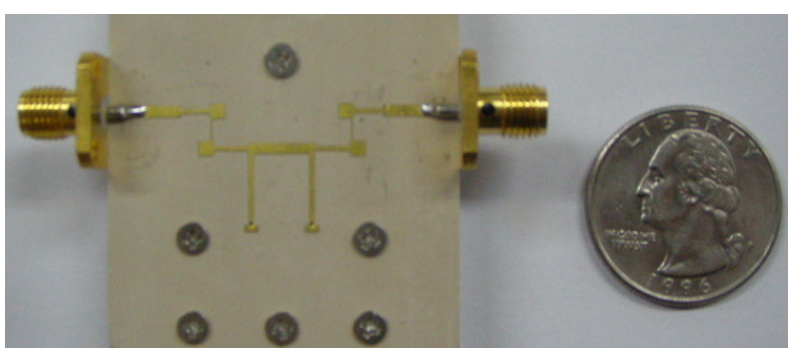

Fig. 10. Photograph of the fabricated UWB bandpass filter.

measured results are in good agreement. However, there is an increase in the insertion loss compared to the simulated result due to the fabrication error. It has a pass-band from $3.1 \mathrm{GHz}$ to $10.6 \mathrm{GHz}$ for a less than 1.7 $\mathrm{dB}$ insertion loss and the return loss is approximately 10 $\mathrm{dB}$ within the pass-band. The most remarkable characteristic is that it has excellent spurious suppression of about $18 \mathrm{~dB}$ in the out-band frequency range and up to

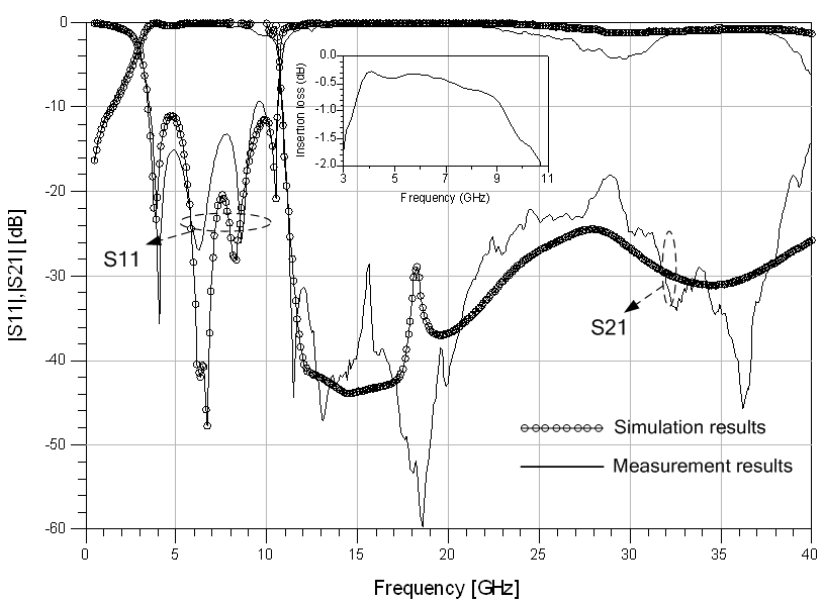

(a)

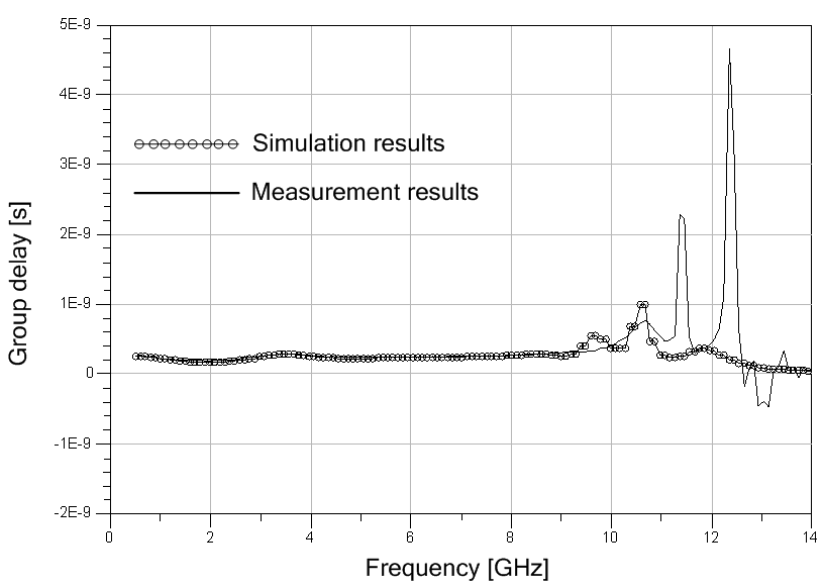

(b)

Fig. 11. (a) Simulated and measured frequency responses. (b) Group delay.

Table 1. Comparison with other wide stop-band UWB band-pass filters

\begin{tabular}{|c|c|c|c|}
\hline & Return loss & Insertion loss & $\begin{array}{c}\text { Spurious } \\
\text { response }\end{array}$ \\
\hline$[11]$ & $15 \mathrm{~dB}<$ & $0.86 \mathrm{~dB}>$ & $\begin{array}{c}\sim 26 \mathrm{GHz} \\
(<-18 \mathrm{~dB})\end{array}$ \\
\hline$[12]$ & $10 \mathrm{~dB}<$ & $1 \mathrm{~dB}>$ & $\begin{array}{c}\sim 25 \mathrm{GHz} \\
(<-20 \mathrm{~dB})\end{array}$ \\
\hline$[13]$ & $10 \mathrm{~dB}<$ & $0.5 \mathrm{~dB}>$ & $\begin{array}{c}\sim 18 \mathrm{GHz} \\
(<-30 \mathrm{~dB})\end{array}$ \\
\hline$[14]$ & $10 \mathrm{~dB}<$ & $0.5 \mathrm{~dB}>$ & $\begin{array}{c}\sim 18 \mathrm{GHz} \\
(<-38 \mathrm{~dB})\end{array}$ \\
\hline This work & $10 \mathrm{~dB}<$ & $1.7 \mathrm{~dB}>$ & $\begin{array}{c}\sim 39 \mathrm{GHz} \\
(<-18 \mathrm{~dB})\end{array}$ \\
\hline
\end{tabular}

$39 \mathrm{GHz}$. The group delay below 1 ns can be observed within the pass-band as shown in Fig. 11(b). Compared to other published results [11] [14], the proposed UWB filter shows a very low spurious response, while the other characteristics are almost the same as in Table 1. 


\section{Conclusion}

We have presented an asymmetrical stepped-impedance low-pass type inverter with an overall electrical length of $\lambda / 4$. In order to improve selectivity, asymmetrical configuration is also discussed. The proposed stepped-impedance low-pass type inverter was analyzed through the even/odd mode analysis, and a 5th-order inverter is designed to be applied to the UWB band-pass filter. As a result, a UWB band-pass filter with an excellent spurious response up to $40 \mathrm{GHz}$ could be achieved. The return loss of the pass-band was also improved. An asymmetrical stepped-impedance low-pass type inverter can be applied to various band-pass filters in which low spurious characteristics are required.

\section{References}

[1] S. -C. Lin, P. -H. Deng, Y. -S. Lin, C. -H. Wang, and C. -H. Chen, "Wide-stopband microstrip bandpass filters using dissimilar quarter-wavelength stepped-impedance resonator," IEEE Trans. Microwave Theory Tech., vol. 54, no. 3, pp. 1011-1018, Mar. 2006.

[2] A. Namsang, T. Majaeng, J. Jantree, S. Chaimool, and P. Akkaraekthalin, "Stepped-impedance hairpin resonators with asymmetric capacitively loaded coupled lines for improved stopband characteristics," IEICE Trans. Electron., vol. e90-c, no. 12, pp. 21852191, Dec. 2007.

[3] M. C. V. Ahumada, J. Martel, and F. Medina, "Parallel coupled microstrip filters with ground-plane aperture for spurious band suppression and enhanced coupling," IEEE Trans. Microwave Theory Tech., vol. 52, no. 3, pp. 1082-1086, Mar. 2004.

[4] J. -T. Kuo, M. Jiang, and H. -J. Chang, "Design of parallel-coupled microstrip filters with suppression of spurious resonances using substrate suspension," IEEE Trans. Microw. Theory Tech., vol. 52, no. 1, pp. 8389, Jan. 2004.

[5] T. Lopetegi, M. A. G. Laso, J. Hernández, M. Bacaicoa, D. Benito, M. J. Garde, M. Sorolla, and M. Guglielmi, "New microstrip 'Wiggly-line' filters with spurious passband suppression," IEEE Trans. Microw. Theory Tech., vol. 49, no. 9, pp. 1593-1598, Sep. 2001.

[6] F. -R. Yang, K. -P. Ma, Y. Qian, and T. Itoh, "A uniplanar compact photonic-bandgap (UC-PBG) structure and its applications for microwave circuits," IEEE Trans. Microwave Theory Tech., vol. 47, no. 8, pp. 1509-1514, Aug. 1999.
[7] J. T. Kuo, E. Shih, "Microstrip stepped impedance resonator bandpass filter with an extended optimal rejection bandwidth," IEEE Trans. Microw. Theory Tech., vol. 51, no. 5, pp. 1554-1559, May 2003.

[8] P. -H. Deng, S. -C. Lin, Y. -S. Lin, C. -H. Wang, and C. H. Chen, "Microstrip bandpass filters with dissimilar resonators for suppression of spurious responses," in Proc. 35th Eur. Microw. Conf., pp. 12631266, 2005.

[9] K. F. Chang, K. W. Tam, "Miniaturized cross-coupled filter with second and third spurious responses suppression," IEEE Microw. Wireless Compon. Lett., vol. 15, no. 2, pp. 122-124, Feb. 2005.

[10] J.-H. Hong, M. J. Lancaster, Microstrip Filters for RF/Microwave Applications, John Wiley \& Sons, Inc., pp. 109-121, 2001.

[11] M. Uhm, K. Kim, and D. S. Filipovic, "Ultra-wideband bandpass filters using quarter-wave shortcircuited shunt stubs and quarter-wave series inverters," IEEE Microw. Wireless Compon. Lett., vol. 18, no. 10, pp. 668-1148, Oct. 2008.

[12] Q. -X. Chu, S. -T. Li, "Compact UWB bandpass filter with improved upper-stopband performance," IEEE Microw. Wireless Compon. Lett., vol. 19, no. 1, Jan. 2009.

[13] K. Li, Y. Yamamoto, D. Kurita, and O. Hashimoto, "An ultra-wideband (UWB) bandpass filter using broadside-coupled structure and lumped-capacitorloaded shunt stub resonators," IEICE Trans. Electron., vol. e90-c, no. 9, pp. 1736-1742, Sep. 2007.

[14] Z. -C. Hao, J.-S. Hong, "Ultra-wideband bandpass filter using multilayer liquid-crystal-polymer technology," IEEE Trans. Microw. Theory Tech., vol. 56, no. 9, pp. 2095-2100, Sep. 2002.

[15] Sai Wai Wong, Lei Zhu, "Implementation of compact UWB bandpass filter with a notch-band," IEEE Microw. Wireless Compon. Lett., vol. 18, no. 1, Jan. 2008.

[16] J. -H. Hong, M. J. Lancaster, Microstrip Filters for RF/Microwave Applications, John Wiley \& Sons, Inc., pp. 109-121, 2001.

[17] L. Zhu, K. Wu, "Short-open calibration technique for field theory-based parameter extraction of lumped elements of planar integrated circuits," IEEE Trans. Microw. Theory Tech., vol. 50, issue 8, pp. 18611869, 2002.

[18] I. -S. Kim, S. -W. Yun, "Compact LPF using asymmetrical microstrip step discontinuity for harmonic suppression," Electronics Letters, vol. 41, no. 16, Aug. 2005. 


\section{Young-Ho Cho}

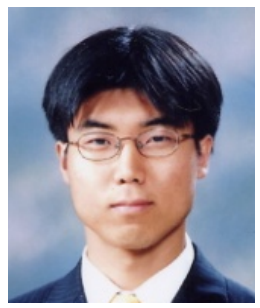

received the B.S. degree in electrical engineering from Sogang University in 2005, Seoul, Republic of Korea. He is currently working toward a Ph.D. degree in electronic engineering at Sogang University. His research interests include synthesizers, RF filters, and RF systems.

\section{Moon-Gyu Choi}

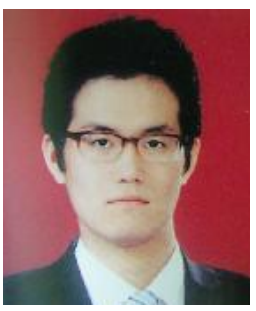

received the B.S. degree in electrical engineering from Sogang University in 2010, Seoul, Republic of Korea. He is currently working toward a M.S. degree in electronic engineering at Sogang University. His research interests include RF filters and power dividers.

\section{Sang-Won Yun}

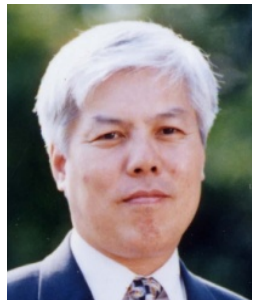

received the B.S. and M.S. degrees in electronic engineering from Seoul National University, Seoul, Republic of Korea in 1977 and 1979, respectively, and a Ph.D. degree in electrical engineering from the University of Texas at Austin in 1984. Since 1984, he has been a professor in the department of electronic engineering, Sogang University, Seoul, Republic of Korea. Since 2010, he has been a project manager in Korea Communications Commission (KCC). From January 1988 to December 1988, he was a visiting professor at the University of Texas at Austin. Dr. Yun was a president of the Korea Institute of Electromagnetic Engineering and Science (KIEES) in 2007. He was a chairman of the IEEE Microwave Theory and Techniques Society (IEEE MTT-S) Korea Chapter. His research interests include microwave and millimeter-wave devices and systems. 